\title{
Urgences
}

\section{L'esprit des lieux : la lettre des textes}

\section{Paul Chanel Malenfant}

Numéro 17-18, octobre 1987

L'esprit des lieux

URI : https://id.erudit.org/iderudit/025423ar

DOI : https://doi.org/10.7202/025423ar

Aller au sommaire du numéro

Éditeur(s)

Urgences

ISSN

0226-9554 (imprimé)

1927-3924 (numérique)

Découvrir la revue

Citer ce document

Malenfant, P. C. (1987). L'esprit des lieux : la lettre des textes. Urgences, (17-18),

85-98. https://doi.org/10.7202/025423ar d'utilisation que vous pouvez consulter en ligne.

https://apropos.erudit.org/fr/usagers/politique-dutilisation/ 


\title{
Paul Chanel Malenfant L'ESPRIT DES LIEUX: LA LETTRE DES TEXTES
}

\section{DES LIEUX VUS}

Tel était, au bout du monde, longuement brumeux à ta saison des pluies, le lieu autour duquel, peu à peu s'érigeait mainte histoire nouvelle.

Jean Ricardou: Révolutions minuscules, p. 167

L'écrivain, le poète qui choisit en tels textes de s'adonner à la rêverie de lieux privilégiés, désigne certes d'abord des référents géographiques: il convoque des réminiscences, réelles ou imaginées, il évoque des atmosphères, des sensations, voire il sait traduire ses paysages en une philosophie ou un art de vivre. Ainsi, Camus, dans ses admirables essais intitulés Noces suivi de L'été, parvient-il à exalter, en regard de maints paysages algériens qui lui sont familiers, cette "tendre indifférence du monde" ainsi que cette énergie de la jouissance cosmique et sensuelle qui fondent sa morale et son esthétique. L'évocation du paysage tient alors de l'hymne, de l'hommage, et le lieu contemplé dans le texte induit celui-ci à l'incantation lyrique:

\begin{abstract}
Au printemps, Tipasa est habitée par les dieux et les dieux parlent dans le soleil et l'odeur des absinthes, la mer cuirassée d'argent, le ciel bleu écru, les ruines ouvertes de fleurs et la lumière à gros bouillons dans les amas de pierres. À certaines heures, la campagne est noire de soleil. Les yeux tentent vainement de saisir autre chose que des gouttes de lumière et de couleurs qui tremblent au bord des cils. L'odeur volumineuse des plantes aromatiques racle la gorge et suffoque dans la chaleur énorme. A peine, au fond du paysage, puis-je voir la masse noire du Chenoua qui prend racine dans les collines autour du village, et s'ébranle d'un rythme sûr et pesant pour aller s'accroupir dans la mer'.
\end{abstract}

Le haut taux d'adjectifs ainsi que l'insistance de la conjonction et de la détermination sont ici trois stratégies syntaxiques qui, définissant et ajoutant les uns aux autres les éléments du décor, signalent donc la description comme fait et effet de langage. Devant l'opacité tangible du paysage, le texte convoque l'amplitude de l'ode ou le foisonnement lié et fondu du tableau impressionniste; tout se passe comme si la sensualité même du lieu imprégnait l'écriture d'une sorte de profusion ou de débordement portés à l'utopique tentative d'une représentation figurative. Les mots consentent ici, devant le réel, à la suggestion ou à l'allusion évocatrices; ils exhibent alors leur état même de langage, état essentiel, distinct de la pulpe ou du grain des choses. 


\section{2. À L'ÉCOUTE DES NOMS DE LIEUXX}

D'autres attitudes d'écriture ou d'appréhension du réel permettent de circonscrire différemment les lieux et de les infléchir dans les textes à titre de véritables "pages paysages", pour adapter ici l'efficace expression empruntée à Jean-Pierre Richard. Ces textes reconnaîtront les toponymes peut-être moins dans leur fonction d'embrayeurs visuels d'un espace circonscrit que dans leur valeur intrinsèque de "mots autonomes" (au sens de Roman Jakobson) et de dispositifs poétiques; les noms de lieu, noms propres et icônes sémantiques de fictions et de remémorations multiples, détiennent alors, tels qu'en eux-mêmes, toutes les possibilités de se constituer en récits et d'activer, aux plans conjoints du contenu et de la structure fictionnelle, les textes où ils adviennent.

Chaque nom a ainsi son spectre sémique, variable dans le temps, selon la chronologie de son lecteur, qui ajoute ou retranche de ses éléments, exactement comme fait la langue dans sa diachronie. Le Nom est en effet catalysable; on peut le remplir, le dilater, combler les interstices de son armature sémique d'une infinité de rajouts.

Cette dilatation sémique du nom propre peut être définie d'une autre façon: chaque nom contient plusieurs «scènes» surgies d'abord d'une manière discontinue, erratique, mais qui ne demandent qu'à se fédérer et à former de la sorte un petit récit, car raconter, ce n'est jamais que lier entre elles, par procès métonymique, un nombre réduit d'unités pleines $^{2}$.

En outre, ces récits virtuels, extensibles et infiniment maniables que sont les toponymes, agiront tour à tour, dans le tissu d'une écriture, en vertu de leur plasticité sémantique, émotive et mnémotechnique, de leur physionomie acoustique ainsi que de leur «corrélation positionnelle" (Tzvetan Todorov) dans une fiction ou un poème.

\subsection{ENTENDRE FLORENCE}

Rappelons ici, à titre de première illustration exemplaire de tel type de posture de poétique toponymique, ce passage où Sartre, dans son Qu'est-ce que la littérature? (1947), procède justement à une mise en fiction (ou sorte de mise en scène cratyléenne) de la ville de Florence, s'adonnant à l'effort d'hybridation vocalique et consonantique du toponyme et s'adressant aux instances biographiques remémorées de son enfance:

Florence est ville et fleur et femme; elle est ville-fleur et ville-femme et fille-fleur tout à fois. Et l'étrange objet qui paraît ainsi possède la liquidité du fleuve, la douce ardeur fauve de l'or et, pour finir, s'abandonne avec 
décence et prolonge indéfiniment par l'affaiblissement continu de l'e muet son épanouissement plein de réserves. À cela s'ajoute l'effort insidieux de la biographie. Pour moi, Florence est aussi une certaine femme, une actrice américaine qui jouait dans les films muets de mon enfance et dont j'ai tout oublié, sauf qu'elle était longue comme un long gant de bal et toujours un peu lasse et toujours chaste, et toujours mariée et incomprise, et que je l'aimais, et qu'elle s'appellait Florence ${ }^{3}$.

Florence préserve certes ici son prestigieux statut de ville d'Italie mais plus encore, et qui importe, le mot qui désigne ce lieu se trouve investi, en vertu de sa configuration sonore, de toute une chaîne sémantique associative: à la connotation florale et fluviale $(F I)$ s'adjoint l'audible matérialité de l'or (Flor) qui illumine le vocable en son centre, jusqu'à l'avènement d'une sorte d'abandon sentimental et confident. Dans sa «décence», Florence fait surgir dans la mémoire du narrateur le souvenir de telle femme alanguie et longue et, par mimétisme syntaxique, longuement déployée en une longue phrase.

À la différence de Camus qui, dans son évocation de Tipasa, s'intéressait, par une pulsion scopique induisant une pulsion érotique, à rendre sensuellement sa vision d'un paysage et d'un lieu, Sartre travaille ici, par une impulsion phonétique qui se mue en saillie biographique, à traduire l'audition d'un nom (de lieu) et à en retracer le retentissement en son histoire personnelle.

\subsection{PROUST: L'ÉCHO DES «NOMS DE PAYS»}

On sait aussi l'importance que Proust a accordée, dans À la recherche du temps perdu, à la stratégie structurante de la nomination:

[Les noms] exaltèrent l'idée que je me faisais de certains lieux de la terre, en les faisant plus particuliers, par conséquent plus réels [...]. Combien ils prirent quelque chose de plus individuel encore, d'être désignés par des noms, des noms qui n'étaient que pour eux, des noms comme en ont les personnes ${ }^{4}$.

En outre, Jean Ricardou a déjà observé comment l'écoute autobiographique de la ville de Florence par Sartre, s'apparentait, en son fonctionnement acoustique et mémorial, à cette autre page célèbre de Proust:

[...] entre Bayeux si haute dans sa noble dentelle rougeâtre et dont le faîte était illuminé par le vieil or de sa dernière syllabe: Vitré dont l'accent aigu losangeait de bois noir le vitrage ancien (...); Quimperlé, lui, mieux attaché, et depuis le moyen âge, entre les ruisseaux dont il gazouille et s'emperle en une grisaille pareille à celle que dessinent, à travers les toiles d'araignées d'une verrière, les rayons de soleil, changés en pointes émoussées d'argent bruni ${ }^{5}$. 
On l'aura entendu: pour Proust comme pour Sartre, l'activité textuelle du toponyme réside peut-être moins en sa valeur géographique référentielle que dans sa condensation signifiante (dans sa mixture conjointe de signifié et de signifiant), dans l'illusion mimétique qu'il autorise, enfin dans ses possibilités d'inflation ou de dissémination phonique et graphique au sein d'un texte. En ce sens, les noms de lieux ou lieux-dits, à l'instar de tout autre motif thématique ou invariant structural, participent de la texture structurante d'une oeuvre; tramés, essaimés dans les textes qu'ils occupent conjointement du point de vue du signifiant et du point de vue du signifié, ils sont des «milieux» propices à la saturation du sens.

Autrement dit, si le Nom [...] est un signe, c'est un signe volumineux, un signe toujours gros d'une épaisseur touffue de sens, qu'aucun usage ne vient réduire, aplatir, contrairement au nom commun, qui ne livre jamais qu'un de ses sens par syntagme [...]. C'est là le prix - ou la rançon - du phénomène $\propto$ d'hypersémanticité » dont il est le siège, et qui l'apparente, bien entendu, de très près au mot poétique ${ }^{6}$.

\subsection{VOIX D'ITALIE}

Cette efficacité du nom propre à s'immiscer dans le grain d'un texte et à y agir, comme subrepticement, par condensation et magnétisme poétiques, voyons encore comment, en instaurant cette brève chronique italienne, elle se réalise dans un poème récent (1983) de Gaston Miron.

Les choses sont loin, les êtres, le monde

toujours plus loin en avant

dans les régions obscures de l'âge

mais un jour vous marchiez près de moi parmi les autres

5. vous avez passé tout ainsi votre main sous mon bras

l'espace de traverser la rue sous la pluie à Padoue

il y a longtemps que ce geste m'était étranger

alors encore une fois trop tard l'éternité m'est revenue

et tout de suite après le désespoir

10. n'oubliez jamais Rossana Carrer la lumière de vos yeux

quand nos regards se sont épris de côté

il y a longtemps que je vous aime à Treviso

ne vous ayant jamais vue

et plus ne vous reverrai??

Quatorze vers: le poème présente donc la structure numérique d'un sonnet mais son découpage syntaxique, oubliant la stratégie rimale et la versification symétrique du modèle, s'effectue plutôt sur le mode de la syncope rythmique en accord avec la thématique de la marche erratique et amoureuse que dit le texte. À la chute des vers 6 et 12 , vers «localisés» en position capitale en regard de la numération du poème, deux noms de villes de la Vénétie: Padoue et Treviso. On 
aura remarqué que la première ville advient sous sa désignation française (Padoue au lieu de Padova) alors que le nom de la seconde (Treviso $=$ Trévise) n'est pas traduit. Certes, à observer les indices narratifs et la fibre acoustique du texte, ce choix n'est pas innocent. En effet, l'audition de Padoue, outre qu'elle condense les pas déjà audibles de la marche qui résonnent dans la "clef allitérative" de "plus", "près», "parmi», "passé», "espace» et "pluie», fait aussi entendre la douceur de la marche et celle de cet étrange geste d'accompagnement ("vous avez passé tout ainsi votre main sous mon bras"), "geste étranger ", maternel peut-être (?), survenu en une ville étrangère qui sera affectueusement nommée ( $P$ Padoue») dans la langue maternelle du narrateur: ceci relevant, donc, du travail de l'expression émotive produite en texte et tramée dans le poème.

Mais de Padoue à Treviso, a-t-on vu le visage de cette énigmatique et sonore Rossana Carrer? Où donc se cache-t-il? Dans «les régions obscures de l'âge" du poète? Dans ce retour de "l'éternité" et du "désespoir»? Plus loin encore. Car «Treviso» (sise au vers 12, lui-même multiple de trois), clandestinement, dissimule trois visions (trois vues) d'un visage absent, absent en ce qu'il est non dit dans le poème et que le poète interpelle avec insistance en ce triptyque terminal au voussoiement poli: «je vous aime» / «vous (...) jamais vue» / "vous reverrai"...

Rossana Carrer: les initiales, précisément, sont celles de "nos regards (...) épris de côté" et ce nom étranger constitue, exactement, une rime de «regard». Rossana Carrer, cet amour anonyme, nommé avec tant d'éclat, n'a donc pas d'autre visage que celui qui se confond en «je vous aime» (à Treviso) et «jamais» (à Padoue). Ainsi donc, une seule fois passera à Padoue, trois fois verra sans voir à Treviso.

Pour Proust, comme le fait remarquer Roland Barthes, "Parme ne désigne pas une ville d'Émilie, située sur le Pô, fondée par les Étrusques, grosse de 138000 habitants; le véritable signifié de ces deux syllabes est composé de deux sèmes: la douceur stendhalienne et le reflet des violettes ${ }^{8}$. De la même manière, pour Miron, les deux villes de Vénétie existent moins à travers leur histoire et leurs paysages que dans les résonnances affectives de leurs noms qui disent, à sons couverts, la fugace aventure d'un amour impossible, d'une "marche à l'amour».

Après la langueur vague de Florence pour Sartre et Rossana Carrer de Miron fuyant de Padoue à Treviso, croira-t-on que toutes les villes italiennes sont femmes et que, sitôt nommées, elles disparaissent, inaccessibles, dans l'aura musicale de leur nom? Pour réfuter 
cette ironique proposition, qu'il me soit permis de faire allusion à la plus magique et sans doute à la plus souvent (d)écrite des villes d'ltalie, à Venise, et d'évoquer une expérience textuelle que j'ai personnellement déjà tentée. Ce qui m'a séduit, justement, à Venise, plus que les clichés des gondoles ou du pont des soupirs, ce sont les lagunes que je me plaisais à rêver comme des langues, langues de mer et langues maternelles. Ainsi, d'une amie, Lise, un jour venue à Venise et dédicataire d'un texte que j'intitulai Lieux dits: italiques (1982), j'écris telles lignes: «Dit-elle, les grandes lèvres ouvertes, si roses ou mûres, ces si belles phrases, bien païennes" ${ }^{9}$. Venise alors m'est aussi devenue femme, et textuelle, et sexuelle, d'où l'investissement libidinal de la suite que j'y écrivis. Vraiment, parce que toute ville est texte, elle conjoint dans le plaisir de sa découverte et de sa nomination à la fois «le plaisir du texte" et "cet obscur objet nommé désir" qu'est aussi le désir d'écrire.

\section{KAMOURASKA: LA MÉMOIRE D'UN TEXTE}

Parfois nanti d'un plus immédiat rendement de sa poéticité, le toponyme peut aussi détenir un rôle expansif d'irradiation dans le tissu d'un texte; alors, "Loin d'appartenir à un élément isolé, le nom "propre» désigne une fonction du récit»" ${ }^{10}$.

Ainsi, dans Kamouraska (1970), roman d'Anne Hébert, le toponyme qui forme l'intitulé constitue-t-il, certes, un important leitmotiv géographique. En effet, l'anse de Kamouraska apparait bien comme le centre paroxystique du drame, par rapport à Québec, Sorel, les États-Unis et toutes les autres distances franchies par les protagonistes de ce roman de la course à la mort, puisqu'elle est, justement, le lieu maudit de l'amour et de la mort, là où pour assouvir sa vengeance et sa passion, George Nelson, l'amant anglophone, va tuer Antoine Tassy, le mari francophone. Mais si le village de Kamouraska culmine, tant par sa force de concentration dramatique que par la fréquence rythmique de sa citation dans la fiction, le toponyme engendre aussi, par diffusion et essaimage sonores, un assez subtil système rimal qui lui confère sa fonction poétique et son rôle d'agent tissulaire dans le texte: «Mon petit Nicolas, fils unique de l'amour. Le sacrifice célébré sur la neige. Dans l'anse de Kamouraska gelé comme un champ sec et poudreux. L'amour meurtrier. L'amour infâme. L'amour funeste. Amour. Amour. Unique vie de ce monde" ". Dans Kamouraska s'entend certes la "contamination pathétique» (Jean Cohen) de l'amour et de la mort qui génère tout le procès diégétique de l'oeuvre, mais aussi l'anagrammatisation approximative de Nicolas, le fils de l'amour, le fils illégitime donc, dissimulé par conséquent dans le lieu même de la mort: Kamou- 
raska. De plus, entre les deux "cas» que constituent l'amant et le mari, pourraient encore s'entendre l'amour comme demande ( «Ask») et la mort comme réponse, cela même qui relève de la fiction romanesque.

L'effraction clandestine du toponyme dans la tessiture du texte s'est effectuée ici par un phénomène d'écholalie entre la toponymie et l'onomastique ${ }^{12}$; le nom du lieu, déjà fort de sa position stratégique de pôle spatial dans le roman, se trouve en quelque sorte surdéterminé, à la fois par le contexte thématique de l'oeuvre et par le nom d'un personnage "en marge" (Nicolas) dont il se souvient. En ce sens, s'il l'on reconnaît encore avec Barthes que «le Nom propre est (...) la forme linguistique de la réminiscence», on admettra que les textes qui, justement, se désignent d'un nom propre seront enclins à se le remémorer, à le "déplier exactement comme on le fait d'un souvenir ${ }^{13}$ dans ses diverses variations sémantiques et parfois jusque dans la filature de la lettre ${ }^{14}$. Ce souvenir que le texte garde de ses noms et qu'il exhibe tour à tour par association lexicales, par connivences ou fragmentations sonores, atteste bien que «Le mot "poétique» est un miroir - brisé» ${ }^{15}$; qu'il est une mémoire qui se disperse dans le texte pour mieux présider à son organisation.

\section{LIEUX D'ÉCRIRE}

De ce travail spéculaire et textuellement fonctionnel du nom propre ou du toponyme, une brève étude de l'incipit de Prochain épisode (1965) d'Hubert Aquin fournira un autre type d'illustration: «Cuba coule en flammes au milieu du lac Léman pendant que je descends au fond des choses. Encaissé dans mes phrases, je glisse, fantôme, dans les eaux névrosées du fleuve et je découvre, dans ma dérive, le dessous des surfaces et l'image renversée des Alpes ${ }^{16}$. Voyons comment, en cet exemple, le rassemblement toponymique inusité ou encore la fabulation géographique attestent de la physionomie et du fonctionnement mêmes d'une écriture. L'association cumulative de Cuba, du lac Léman, du fleuve Saint-Laurent et des Alpes, si elle peut s'entendre comme une aberration géographique, relève surtout d'une adroite confusion, propice à l'établissement d'un non-lieu ou d'un éclectique no man's land, lequel, pour n'être logiquement admissible que dans la dérive du rêve ou de la mémoire, serait sans doute significatif dans la perspective de «l'impossible pays" que décrit le roman. Plus encore, le détournement référentiel des lieux, qui les oblitère en tant qu'indices géographiques, s'assortit ausi d'une distorsion spatiale où se conjoignent le naufrage vertical de Cuba ( Cuba coule...») et le naufrage horizontal du narrateur ( «je glisse... dans ma dérive...»); jusqu'à ce qu'une lecture de l'articulation 
symétrique des phrases (ces phrases fluviales et névrotiques d'un roman fleuve?) vienne à en montrer les réseaux d'équivalences, la circularité syntaxique et, par conséquent, le vertige réverbérant:

au milieu/au fond

dans mes phrases/dans les eaux/dans ma dérive encaissé/névrosées/renversée

flammes/fond/phrases/fleuve/surfaces

le dessous des surfaces

\section{l'image renversée des Alpes: g/p}

Certaines proximités phonémiques et aimantations sémantiques disent ici le malaise conjoint d'un éc(r)oulement et d'une descente, d'un encaissement et d'un renversement, bref toute une dynamique de «l'en dedans» attestée par la fréquence, que nous avons déjà soulignée, des occurrences du son "an» et par le répertoire des dispositifs d'inclusion: "au milieu", "au fond", "dans" (3 fois), "le dessous". L'organisation de la redondance expressive et d'une certaine métrique du texte affichent un en-dessous de la signification ou, sous le sens de surface ("le dessous des surfaces»), d'autres strates archéologiques de sens; tout se passe comme si la "vue» des lieux, leur impossible rassemblement ailleurs que dans l'enfermement de la phrase et de l'imaginaire, le "parcours" erratique et fantasmatique qui conduit de Cuba aux Alpes et au lac Léman, induisaient, par procédure mimologique, l'exercice d'un "point de vue" ou d'un "cours" (d'un courant) sur la forclusion d'une écriture. Tant il est vrai que selon Cratyle «la vertu des noms est d'enseigner» et qu' «il y a une propédeutique des noms, qui conduit, par des chemins souvent longs, variés, détournés, à l'essence des choses» ${ }^{17}$.

D'ailleurs, chez Aquin, à l'encerclement suffocant et au désordre sinistré des lieux extérieurs, s'adjoindront l'agressivité et la clôture des «espaces du dedans» qui, à leur tour, thématiseront la pratique de l'écriture: "J'écris sur une table à jeu, près d'une fenêtre qui me découvre un parc cintré par une grille coupante qui marque la frontière entre l'imprévisible et l'enfermé» ${ }^{18}$. Circuit ininterrompu qui conduira le roman jusqu'a'son impossible dénouement, jusqu'au mot FIN, puisque la page, comme tout paysage et comme tout espace, est à la fois une prison et une hallucination.

Le collage géographique inusité et tout à fait baroque qui préside à la constitution du paysage aquinien, l'anarchie délibérée de la prolifération toponymique qui désoriente le texte, de même que les 
nombreux voyages (de Montréal à Genève) et trajectoires de toutes sortes (de l'Hôtel d'Angleterre au Château de Coppet) qu'effectuera le narrateur, disent le perpétuel déplacement d'une écriture baroque, donc toujours disponible à la métamorphose et aux prises avec la prolifération des miroirs: "relation ou journal, récit ou rêverie, tout déplacement devenu écriture est un regard, une lecture ou un pélerinage ${ }^{19}$. Dans cette oeuvre, la juxtaposition fantasmatique des lieux raconte ce non-lieu psychique du sujet écrivant tant il est vrai, comme le rappelle Bernard Beugnot, que «tout paysage est état d'âme, toute ville, plus encore si elle est chargée d'histoire, est déjà texte, c'est-à-dire signe, hiéroglyphe, symbole ${ }^{20}$. En ce sens, les lieux de Prochain épisode constituent bien les figures métaphoriques d'un texte (et d'un projet de texte) en marche continuelle. Récit de voyage, donc, et voyage incessant d'une écriture: écriture itinérante qui se refuse à l'état d'arrêt.

Ce parallélisme entre l'investissement toponyme ou la description concertée de tel paysage et la pulsion scripturale s'effectuera, en d'autres textes, sous le mode de la représentation mimétique: le lieu décrit fusionnera la procédure même de l'écriture, la description locale se doublera d'une désignation scripturale. En ce sens, comparons encore les incipit de deux romans, celui des Lieux-dits. Petit guide d'un voyage dans le livre (1969) de Jean Ricardou (on notera comment ce titre est particulièrement intéressant en regard de notre propos) et celui des Fous de bassan (1982) d'Anne Hébert. En ces deux textes, pourtant très différents dans leur travail de production textuelle, l'entrée en matière conjoint, de façon analogue, la description d'un paysage et le procès d'écriture.

\title{
De Jean Ricardou:
}

\author{
Bannière \\ À peine franchie, sous les nuées, cette sombre ligne de \\ faîte, tout le pays, en contrebas, dispense des reflets. \\ Selon une perspective plongeante qui minimise les \\ ondulations du sol, la vallée de Bannière offre au \\ voyageur sa sinuosité insensible ${ }^{2 !}$.
}

\section{D'Anne Hébert:}

La barre étale de la mer, blanche à perte de vue, sur le ciel gris, la masse noire des arbres, en ligne parallèle derrière nous ${ }^{22}$.

Observons d'abord comment, chez Ricardou, le pays de Bannière constitue le titre ('e nom propre) du texte et qu'il fait jouer polysémiquement, par sa position centrale au haut de celui-ci, sa fonction d'enseigne ou d'étendard placé sur l'espace de la page. En 
outre, dans le contexte des Lieux-dits, "les nuées» qui apparaissent sous la «bannière" renvoient à la surface blanche qui précède son inscription. On retrouve d'ailleurs, chez Ricardou, dans une nouvelle intitulée "Plage blanche», cette reconnaissance de la page blanche et de la plage blanche comme lieux scriptibles et lisibles, comme instances monochromes entre l'écriture et la lecture: "Qu'un espace blanc si possible, alentour, [les] inscrive au contraire, à chaque fois, en des lieux bien lisibles. [...] Si bien que, sauf improbable découverte ultérieure, subsiste seule, en ce lieu, maintenant, ensevelie par l'ombre, toute une marge blanche ${ }^{23}$.

De la même manière, "cette sombre ligne de faîte" qui apparaît «sous les nuées» figure à la fois la ligne de l'horizon et la première ligne (tracée) du texte placée juste au dessus d'elle. Sur cette lancée, "tout le pays», à savoir tout le texte ou tout le lieu qui s'apprête à se dire ou à s'écrire, va se constituer en une prolifique "population des miroirs", en une invasion de "reflets» et d'effets spéculaires. Le "voyageur", le lecteur, muni du "guide" qu'il tient actuellement en main, n'a plus qu'à consentir à cette «perspective plongeante» (à cette plongée dans l'écriture) et à s'engager dans «la vallée de Bannière», ce paysage, cette page, ce livre.

Plus discrètement motivé, l'incipit du roman d'Anne Hébert inscrit lui aussi le paysage comme espace littéraire. En effet, la première ligne du texte - «La barre étale de la mer, blanche à perte de vue,..." - outre qu'elle est précédée de la conventionnelle surface blanche, se désigne à la fois comme ligne de mer horizontale et comme tracé descriptif. Cette mer, dont on sait qu'elle va constituer l'espace dramatique le plus important du roman, dès sa nomination, se thématise en texte par allusions métaphoriques. Ainsi, l'étendue marine convoque, en son étalement, la page blanche, au même titre que "la masse noire des arbres, en ligne parallèle derrière nous" sollicite sur celle-ci l'alignement ponctuel des signes typographiques. lci, le champ sémantique de la description d'une part, la symétrie syntaxique de la phrase nominale qui s'assortit à l'ordonnance du paysage décrit d'autre part contribuent à mimer la genèse d'une inscription. "Ce qui s'allégorise alors, dans cette relation du récit au paysage, c'est peut-être bien la naissance de la fiction $»^{24}$. Car la scénographie du paysage participe de la géographie même du texte qu'on peut ainsi disposer:

La barre étale de la mer,

blanche,

à perte de vue,

sur le ciel gris, 
la masse noire des arbres,

en ligne parallèle derrière nous.

blanche / gris / noire

La barre / la masse / en ligne parallèle

Une fois encore, la vue d'un lieu induit la venue d'un livre ${ }^{25}$, tant il est vrai que «Voir ouvre tout l'espace au désir, mais voir ne suffit pas au désir ${ }^{26}$ : comme si voir était, en quelque sorte, l'acte prémonitoire et générateur de toute histoire.

\section{DES LIEUX PRODUITS}

Sans dénier, certes, les possibles efficaces textuelles d'une rêverie contemplative des lieux et les émanations spiritualistes, voire l'évocation d'états d'âme, de souvenirs, de constructions imaginaires que peut faire surgir la seule profération de leurs noms, je me suis surtout attardé ici à l'observation de textes où les lieux et leurs noms agissent davantage à titre de mots surcodés, de récits potentiels, et où ils s'investissent en tant qu'éléments fonctionnels, mécaniques et synesthésiques, dans la fabrique même d'une écriture. Ce parti pris relève, bien sûr, d'une attitude de lecture qui se veut davantage attentive aux stratégies de production textuelle qu'aux effets d'expression engagés dans tel texte par la description ou la nomination des lieux. En outre, on sait comment la caractérisation des textes par les lieux qu'il s'approprient est désormais vulnérable et comment les désignations thématiques comme "poésie du pays», "roman de la terre", "roman de la ville" ne rendent pas forcément compte de la manière dont ces oeuvres produisent leurs lieux. De plus, si l'on considère toutes ces opérations de mises en abyme et de récitsgigognes, exploitées par le roman moderne, on constate qu'elles tendent à préférer à la station descriptive le déplacement multiplicateur de l'espace et du temps, pour dire, structuralement, l'ubiquité des lieux et des histoires. Et même la description hyperréaliste des lieux et des choses, exploitée par tant d'oeuvres contemporaires, dans Le planétarium de Nathalie Sarraute ou dans La jalousie d'Alain Robbe-Grillet par exemple, tend sans doute moins à figer la représentation figurative ou photographique qu'à souligner, avec réalisme, l'illusion référentielle entre le réel et le langage. C'est bien pourquoi, dans Le parti pris des choses de Francis Ponge, "Les plaisirs de la porte", "La crevette" ou les "Bords de mer" sont, d'abord et avant tout, des textes. Enfin, songeons à l'importance, dans tant d'oeuvres québécoises contemporaires (d'Agonie de Jacques Brault à La vie en prose et à La constellation du cygne de Yolande Villemaire), de la prolifération des lieux, comme s'il s'agissait, pour ces textes, d'orien- 
ter l'écriture en désorientant la géographie, de détourner à la fois la fixité d'une appartenance locale et la faction descriptive d'une écriture.

En regard de toutes ces procédures de détournement, dans les textes du lieu comme folklore ou comme inaliénable patrie, il me semble que si, très certainement, les lieux nous créent, ce sont les oeuvres qui les recréent pour nous les rendre dans leur plus juste mesure et leur plus stricte intimité.

\title{
6. PAYS D'ENFANCE
}

Ainsi, mon intérêt pour l'impact quasi physiologique qu'exerce en littérature - et, par conséquent, chez le lecteur - la désignation toponymique sous toutes ses formes relève du fait que «l'esprit des lieux», justement, s'immisce jusque dans la lettre des textes et qu'il les infléchit, matériellement, comme sytème dynamique de signification. Le genus loci agit bien comme instance génétique de l'écriture parce qu'il est d'abord et avant tout une perception physique et mnémotechnique de l'espace et du pays. Dans cette perspective, je reconnais avec Barthes que:

\begin{abstract}
«lire» un pays, c'est d'abord le percevoir selon le corps et la mémoire, selon la mémoire du corps. Je crois que c'est à ce vestibule du savoir et de l'analyse qu'est assigné l'écrivain: plus conscient que compétent, conscient des interstices mêmes de la compétence. C'est pourquoi l'enfance est la voie royale par laquelle nous connaissons le mieux un pays. Au fond, il n'est pays que de l'enfance ${ }^{27}$.
\end{abstract}

Dès lors, retracer l'esprit des lieux procéderait d'un retour à l'état d'enfance, là où dans l'apprentissage de la langue première et du travail de l'écriture, les choses et les paysages préservent cette substantielle connivence et cette concrète solidarité avec les noms et les mots. Ainsi croyait Tinamer de Portanqueu pour qui, dans L'amélanchier (1970) de Jacques Ferron, le Mont-Thabor délimitait le bon et le mauvais côté des choses, Tinamer qui dira, comme faisant écho à l'assertion barthésienne:

Mon enfance fut fantasque mais sédentaire de sorte qu'elle subsiste autant par ma mémoire que par la topographie des lieux où je l'ai passée, en moi et hors de moi. Je ne saurais me dissocier de ces lieux sans perdre une part de moi-même ${ }^{28}$.

\section{NOTES}

1. Albert Camus: Noces suivi de L’été, coll. cLe livre de poche», Paris, Gallimard, 1967, p. 13.

2. Roland Barthes: ‘Proust et les noms*, dans Le degré zéro de l'écriture, coll. «Points*, Paris, Seuil, 1972, p. 127.

3. Jean-Paul Sartre: Qu'est-ce que la littérature?, coll. ildées*, Paris, Gallimard, 1969, p. 21.

4. Marcel Proust: Du còté de chez Swann, dans À la recherche du temps perdu, coll. *Bibliothèque de la Pléiade*,

Pléiade I, Paris, Gallimard, p. 387. 
5. Marcel Proust: Ibid, p. 388-389. Cité dans Jean Ricardou, Pour une théorie du nouveau roman, Paris, Seuil, 1971,

p. 10 .

6. Roland Barthes: Ibid. p. 125-126.

7. Gaston Miron: [Sans titre), dans Estuaire. Montréal, no 30, hiver 1984, p. 80.

8. Roland Barthes: Ibid, p. 127.

9. Paul Chanel Malenfant: Les noms du père suivi de Lieux dits: italiques, Montréal, Éditions du Noroît, 1985, p. 88. Perspicacité d'une lectrice (Christiane Asselin, dans Urgences, Rimouski, no 14, août 1986, p. 76) qui a déjà fort bien remarqué les connotations biographiques et pulsionnelles de ce méme passage:

Dédié à Lise Guèvremont, le second volet du recueil s'ouvre sur des Lieux dits: italiques. Halique, parce qu'ttalie bien sür, parce que Venise surtout, photos à l'appui. ttalique aussi comme mouvement du mot «déformé* (p. 78), comme mouvement des eaux. En ces lieux dits c'est tout le texte qui se trowve en position insulaire, qui émerge du silence pour se conjoindre au sexe, et dire «les grandes lèvres bien ouvertes, si roses ou múres, ces si belles phrases bien paiennes" ( $p$. 88 ), car le moment de l'écriture-texte reproduit, dans toute sa prof-kondeur., le sexe de l'écriture. *Venise, et «lèvres* ne sont-ils pas, anagrammes partiels, dans les prénom et nom de la dédicataire?

10. Jean Ricardou: Pour une thérie du nouveau roman, p. 52.

11. Anne Hébert Kamouraska, Paris, Seuil, 1970, p. 11.

12. Gérard Genette (*Matière de Venise *, dans Territoires de l'imaginaire. Pour Jean-Pierre Richard, Paris, Seuil, 1986, p. 73-84) a observé, chez Proust, un phénomène très ample et très structurant dinterférence entre la toponymie et l'onomastique. En effet, analysant une page d'A la recherche du temps perdu qui, initialement ouvrait Un amour de Swann et que Proust a reportée dans La fugitive. Genette montse comment al'espace Verdurin* est conjoint à al'espace vénitien s; de plus, le déplacement de cette page, entre autres, aurait permis à Proust d'assortir plus étroitement, à la conformation mème de Venise, l'architecture d'A la recherche du temps perdu. C'est en ce sens que Genette écrit: *La construction en patchwork, le bricolage textuel sont ici à leur comble, comme si Proust avait voulu y accorder fidèlement l'écriture à son objet, et mimer par un texte en puzzle le désordre labyrintrique et délicieusement déconcertant d'une ville emblématique, entre toutes, de sa prédilection- (p. 83-84).

13. Roland Barthes: Ibid., p. 124.

14. À titre d'exemple de *lettres filées*, et comme illustration de la connivence acoustique que Proust a instaurée entre les personnages et les lieux d'A la recherche du temps perdu, on lira avec intérêt le livre d'Alain Roger: Proust. Les plaisirs et les noms, coll. * L'infini *, Paris, Denoël, 1984. Je cite cet extrait particulièrement convaincant (p. 132): $J$ 'aime à croire, d'ailleurs, que Proust a transformé Bolbec (non loin du Havre) en Balbec, pour qu'il puisse accueillir Albertine, comme son lieu naturel; de méme que, selon Brichot. *Balbec est probablement une corruption de Dalbec* (II, 936), et des plus opportunes, puisqu'elle permet d’associer à Balbec, mais aussi l'une à lautre, celles qui, *au coeur* du narrateur. resteront à jamais attachées, et qu'il s'accusera d'avoir assassinées. Albertine et Bathilde. prénom de sa grand-mère. (...) Quoi qu'il en soit, la symbiose est si forte entre le «nom de pays* et le *nom de personnes* - *mes désirs de Balbec avaient si bien múri le corps d'Albertine* (II,387) - que le narrateur se demandera si le *désir * d'Albertine n'était pas «lui-mèrne une forme paresseuse, lâche et incomplète de posséder Balbec. (II,351).

15. Gérard Genette: Mimologiques. Voyage en Cratylie, coll. *Poétique*, Paris, Seuil, 1976, p. 302.

16. Hubert Aquin: Prochain épisode. Montréal, Le cercle du livre de France, 1978, p. 7.

17. Roland Barthes: Ibid., p. 133-134.

18. Hubert Aquin: Ibid., p. 7. Cette phrase suit immédiatement les deux précédentes, déjà citées.

19. Bernard Beugnot: «Les soupirs de la mémoire: Venises tex/tuelles», dans Trois, Laval, vol. 2, no 1, septembre 1986, p. 5. Voir aussi chez Roland Barthes: *La ville est un idéogramme: le texte continuen, dans L'empire des signes, coll. "Champs*, Genève et Paris, Les sentiers de la création, Skira et Flammarion, p. 44.

20. Bemard Beugnot: tbld., p. 5.

21. Jean Ricardou: Les lieux-dits. Pett guide d'un voyage dans le Ilvre, coll. * 10/18, Paris, Galljmard, 1972, p. 11.

22. Anne Hébert: Les fous de bassan, Paris, Seuil, 1982, p. 13.

23. Jean Ricardou: *Plage blanche*, dans Révolutions minuscules, coll. *Le chemin*, Paris, Gallimard, 1971, p. 154-155.

24. Jeannine Jallat: ‘Le paysage et sa fiction. Pour un imaginaire du lieu romanesques, dans Teritoires de limaginaire. Pour Jean-Pierre Richard, Paris, Seuil, 1986, p. 94

25. Voyons encore comment, par cet exemple systématique emprunté à Jean Ricardou («Autobiographie*, dans Révolutions minuscules, coll. aLe chemin *, Paris, Gallimard, 1971, p. 164-165), les lieux se transforment tour à tour en personnages, en livres, en histoires:

Au nord de la Russie, la presqu'île de Kanon forme une téte étrangement casquée, dont, à n'en pas douter, le proll considère l'anfractueuse mer Blançhe. À l'ouest, où le soir Yénus vient s'inscrire il y a avec la Baltique, une femme à genoux et qui lit. Les épaules et le visage sont le golfe de Botnie: son livre le golfe de Finlande. $\mathrm{Si}$, au-delà de la mer Blanche, on prolonge le regard du guerrier étrange il vient se poser sur le lture de la femme agenouillée. Et sit, par-delà les feuillets, le regard de cette femme se continue lui-mème encore à travers toute l'épaisseur du continent et la presqu'ile de Crimée, c'est la mer Noire qu'tl vient rejoindre. Loin de me paraitre contingent, ce croisement et ces inversions formaient le départ de toutes manières de rêveries. J'en déduisais par exemple que la lecture suppose un dédoublement parfait: rencontrant la blancheur et le noir, le papier et l'encre, il était alors possible de suivre tel récit peu à peu écrit en lignes alternées sur la page.

En outre, Il suffit de prolonger l'axe visuel du guerrier au-delà du ltvre liquide pour que, bien après tout Etat balte, Venise à son tour soit atteinte, et la Corse. Comme la ligne féminine qui le croise rencontre de son côté l'Asie en Turquie, puis en Palestine, et l'Afrique en Haute-Égypte, c'est une 
byzantine histoire, peu à peu, qui s'inscrivait en moi. Récit invraisemblable en lequel se trouvait prise, inverse Asie, une Isa nécessaire.

Le *il» et *lile* constituent un fil qui se tisse en un *lit* et en un *livre*; on connait aussi l'efficace de la *Plage blanche* chez Ricardou. Dans cette mème perspective on pourrait évoquer les métaphores de la page/plage et des mots/flots dans tel poème de Roland Giguère, ses mots-flots*, Les armes blanches (1954) dans L'áge de la parole, coll. «Rétrospectives*, Montréal, Edition de l'Hexagone, 1965, p. 107.

26. Jean Starobinski: L'oell vivant, coll. Le chemin., Paris, Gallimard, 1968, p. 13

27. Roland Barthes: Incidents, Paris, Sevil, 1987.

28. Jacques Ferron: L'amélanchier, Montréal, Vb éditeur, 1978, p. 11. 